\title{
Rheumatology in East Asia
}

\author{
Kazuhiko Yamamoto ${ }^{1 *}$, Yeong-Wook Song ${ }^{2}$ and Zhan-guo Li
}

\begin{abstract}
Europe and North America have been leaders in rheumatology for many years. However, for more than a decade now the East Asian region has been catching up dramatically. Some aspects of rheumatology in East Asia are now almost comparable to those in the European League Against Rheumatism (EULAR) and the American College of Rheumatology (ACR). In this article, we describe recent progress in rheumatology in East Asia, focusing specifically on Japan, Korea, and China.
\end{abstract}

Keywords: Japan, Korea, China, rheumatology, JCR, $K C R, C R A, A P L A R$, EAGOR

\section{Background}

This manuscript aims to contribute to the series entitled 'Rheumatology around the world'.

\section{General situation of rheumatology in East Asia} Japan is one of the four countries that established the South East Asia and Pacific Area League Against Rheumatism in 1963, which has now been renamed the Asia Pacific League of Association for Rheumatology (APLAR). The Japan College of Rheumatology (JCR) has nearly 10,000 members and about 4500 registered rheumatology specialists. More than 6000 attend each annual meeting of the JCR.

In South Korea, the first rheumatology symposium was held in 1981. Thereafter, in 1992, a new subspecialty board system was established. The rheumatologists certified by the Korean College of Rheumatology (KCR) has increased to more than 300. Similarly, Chinese rheumatology has developed rapidly over the past 20 years. In 2008, about 2600 rheumatologists were reported by the Chinese Rheumatology Association (CRA) and, in 2014, this figure had risen to over 5000 rheumatologists.

\footnotetext{
* Correspondence: kazuhiko.yamamoto@riken.jp

${ }^{1}$ RIKEN Center for Integrative Medical Sciences, 1-7-22 Suehiro-cho,

Tsurumi-ku, Yokohama, Kanagawa 230-0045, Japan

Full list of author information is available at the end of the article
}

Apart from their domestic rheumatology meetings, APLAR meetings have recently been held annually and have become important opportunities to sustain communication and discussion. Furthermore, in 1997 the KoreaJapan Combined Meeting of Rheumatology (KJCMR) was started which was then expanded to include China as the East Asia Group of Rheumatology (EAGOR) in 2005. In this way, rheumatologists in East Asia have been getting together to communicate and improve themselves through friendly rivalry.

\section{Clinical aspects of rheumatology}

Pharmaceutical administration and regulations in Japan were rather conservative. For example, methotrexate (MTX) was approved for rheumatoid arthritis (RA) in 1999, which was more than 10 years later than in Western countries. However, the system in Japan has improved, and approval intervals have been shortened over the past several years. One of the most characteristic administrative policies on biological therapies in Japan is compulsory investigation of all cases treated with a newly approved biological drug. According to this regulation, all RA patients, several thousand to nearly 10,000 , who received an original biological drug were registered and safety data for the initial 6 months were reported. These procedures have strengthened clinical rheumatology in Japan. Several important clinical findings were reported from studies during and after these clinical investigations [1].

Anti-tumor necrosis factor (TNF) biologic treatment for RA was started in Korea in 2000. In 2011, the KCR Biologic Registry (KOBIO) was launched to monitor clinical effects, as well as adverse events, in patients treated with biologic agents.

On the other hand, the CRA published management guidelines for 23 rheumatic diseases to direct practice in China. However, there still exists heterogeneity in clinical practice.

\section{Basic research in rheumatology}

Japan has been one of the leaders in basic immunology, with discoveries of several cytokines such as invterleukin 
(IL)-6 [2], regulatory T cells, and signals in innate immunity. On the other hand, clinical immunology including rheumatology research has not been as strong as basic immunology, although interesting findings have been regularly published from Japan.

Since 2000, Korean rheumatologists have joined innovative government research projects. Several important findings have been published, including antigen-specific $\mathrm{T}$ cells in RA and synovial cells [3]. The osmoprotective transcription factor nuclear factor of activated $\mathrm{T}$ cells (NFAT)5, which regulates macrophage survival by inducing CCL2 secretion, was found to enhance chronic arthritis by conferring apoptotic resistance to activated macrophages [4]. Copy number of leukocyte-specific protein (LSP) 1 was significantly lower in RA patients suggesting its role in the pathogenesis of RA by promoting migration of $\mathrm{T}$ cells into the target tissues [5].

Chinese rheumatologists have been also contributing greatly. For example, TNF-induced FOXP3 dephosphorylation was reported in regulatory $\mathrm{T}$ cells in RA [6]. He et al. reported a novel circulating CCR7 ${ }^{\mathrm{lo}} \mathrm{PD}-1^{\mathrm{hi}}$ follicular helper T cell (Tfh), which was correlated with clinical indices and autoantibody production in RA and systemic lupus erythematosus (SLE) [7]. Zhu et al. showed that IL-17 contributes to autoimmune pathogenesis by suppressing miR-23b expression in resident cells and promoting proinflammatory cytokine expression in patients with SLE and RA [8]. A study showed that the oral and gut microbiomes were perturbed in RA and were partly normalized after treatment [9]. Furthermore, treatment with low-dose recombinant human IL-2 was reported to selectively modulate the numbers of regulatory $\mathrm{T}$ cells, Tfhs, and IL-17-producing helper T cells with marked reductions of disease activity of SLE [10].

Genetic studies in rheumatology in East Asia are rather advanced. Before the current genome-wide association studies (GWAS), several leading GWASs were published from Japan [11, 12]. Furthermore, researchers in East Asia joined the project of transethnic meta-analysis of RA GWASs [13], and important papers were subsequently published from Japan, Korea, and China. Studies of SLE and Behçet's disease have also been extensively performed. One GWAS in the Han population identified two new susceptibility loci for ankylosing spondylitis [14]. In addition, a GWAS in Han Chinese identified a susceptibility locus for primary Sjögren's syndrome at 7q11.23 [15]. Thus, strong genetic studies are now conducted in this region. Moreover, functional genomic and epigenetic studies have also been conducted by several researchers in this region [16, 17].

In conclusion, rheumatology in East Asia has been dramatically developing in several fields in both clinical and basic aspects.

\section{Abbreviations}

ACR: American College of Rheumatology; APLAR: Asia Pacific League of Association for Rheumatology; CCL: C-C motif chemokine ligand; CCR: C-C motif chemokine receptor; CRA: Chinese Rheumatology Association;

EAGOR: East Asia Group of Rheumatology; EULAR: European League Against Rheumatism; FOXP3: Forkhead box P3; GWAS: Genome-wide association studies; IL: Interleukin; JCR: Japan College of Rheumatology; KCR: Korean College of Rheumatology; KJCMR: Korea-Japan Combined Meeting of Rheumatology; KOBIO: Korean College of Rheumatology Biologic Registry; LSP: Leukocyte-specific protein; miR: MicroRNA; MTX: Methotrexate; NFAT: Nuclear factor of activated T cells; PD: Programmed cell death; RA: Rheumatoid arthritis; SLE: Systemic lupus erythematosus; Tfh: Follicular helper T cells; TNF: Tumor necrosis factor

\section{Acknowledgements}

Not applicable.

Funding

There is no funding regarding this article.

Availability of data and materials

Data sharing is not applicable to this article as no datasets were generated or analyzed.

\section{Authors' contributions}

KY wrote the main part of the manuscript. After reading the initial manuscript, YWS and ZL added several sentences. All the authors then read and approved the final manuscript.

Ethics approval and consent to participate

Not applicable.

Consent for publication

Not applicable.

\section{Competing interests}

The authors declare that they have no competing interests.

\section{Publisher's Note}

Springer Nature remains neutral with regard to jurisdictional claims in published maps and institutional affiliations.

\section{Author details}

${ }^{1}$ RIKEN Center for Integrative Medical Sciences, 1-7-22 Suehiro-cho, Tsurumi-ku, Yokohama, Kanagawa 230-0045, Japan. ${ }^{2}$ Seoul National University Medical Research Center, Seoul, South Korea. ${ }^{3}$ Peking University People's Hospital, Peking, China.

Published online: 23 March 2018

\section{References}

1. Takeuchi T, Tatsuki Y, Nogami Y, Ishiguro N, Yamanaka H, et al. Postmarketing surveillance of the safety profile of infliximab in 5000 Japanese patients with rheumatoid arthritis. Ann Rheum Dis. 2008;67:189-94.

2. Hirano T, Yasukawa K, Harada H, Taga T, Watanabe $Y$, Matsuda T, et al, Complementary DNA for a novel human interleukin (BSF-2) that induces $B$ lymphocytes to produce immunoglobulin. Nature. 1986;324:73-6.

3. Yoo SA, You S, Yoon HJ, Kim DH, Kim HS, Lee K, et al. A novel pathogenic role of the ER chaperone GRP78/BiP in rheumatoid arthritis. J Exp Med. 2012;209:871-86.

4. Choi S, You S, Kim D, Choi SY, Kwon HM, Kim HS, et al. Transcription factor NFAT5 promotes macrophage survival in rheumatoid arthritis. J Clin Invest. 2017;127:954-69.

5. Hwang SH, Jung SH, Lee S, Choi S, Yoo SA, Park JH, et al. Leukocyte-specific protein 1 regulates T-cell migration in rheumatoid arthritis. Proc Natl Acad Sci U S A. 2015;112:E6535-43.

6. Nie H, Zheng Y, Li R, Guo TB, He D, Fang $L$, et al. Phosphorylation of FOXP3 controls regulatory $T$ cell function and is inhibited by TNF- $a$ in rheumatoid arthritis. Nat Med. 2013;19:322-8. 
7. He J, Tsai LM, Leong YA, Hu X, Ma CS, Chevalier N, et al. Circulating precursor CCR7(lo)PD-1(hi) CXCR5 ${ }^{+} \mathrm{CD4}^{+} \mathrm{T}$ cells indicate Th cell activity and promote antibody responses upon antigen reexposure. Immunity. 2013;39:770-81.

8. Zhu S, Pan W, Song X, Liu Y, Shao X, Tang Y, et al. The microRNA miR-23b suppresses IL-17-associated autoimmune inflammation by targeting TAB2, TAB3 and IKK-a. Nat Med. 2012;18:1077-86.

9. Zhang X, Zhang D, Jia H, Feng Q, Wang D, Liang D, et al. The oral and gut microbiomes are perturbed in rheumatoid arthritis and partly normalized after treatment. Nat Med. 2015;21:895-905.

10. He J, Zhang X, Wei Y, Sun X, Chen Y, Deng J, et al. Low-dose interleukin-2 treatment selectively modulates CD4(+) T cell subsets in patients with systemic lupus erythematosus. Nat Med. 2016;22:991-3.

11. Suzuki A, Yamada R, Chang X, Tokuhiro S, Sawada T, Suzuki M, et al. Functional haplotypes of PADI4, encoding citrullinating enzyme peptidylarginine deiminase 4, are associated with rheumatoid arthritis. Nat Genet. 2003;34:395-402.

12. Kochi Y, Yamada R, Suzuki A, Harley JB, Shirasawa S, Sawada T, et al. A functional variant in FCRL3, encoding Fc receptor-like 3, is associated with rheumatoid arthritis and several autoimmunities. Nat Genet. 2005;37:478-85.

13. Okada Y, Wu D, Trynka G, Raj T, Terao C, Ikari K, et al. Genetics of rheumatoid arthritis contributes to biology and drug discovery. Nature. 2014:506:376-81.

14. Lin Z, Bei JX, Shen M, Li Q, Liao Z, Zhang Y, et al. A genome-wide association study in Han Chinese identifies new susceptibility loci for ankylosing spondylitis. Nat Genet. 2012;44:73-7.

15. Li Y, Zhang K, Chen H, Sun F, Xu J, Wu Z, et al. A genome-wide association study in Han Chinese identifies a susceptibility locus for primary Sjögren's syndrome at 7q11.23. Nat Genet. 2013:45:1361-5.

16. Shen N, Liang D, Tang Y, de Vries N, Tak PP. MicroRNAs-novel regulators of systemic lupus erythematosus pathogenesis. Nat Rev Rheumatol. 2012;8: 701-9.

17. Ishigaki K, Kochi Y, Suzuki A, Tsuchida H, Tsuchiya H, Sumitomo S, et al. Polygenic burdens on cell-specific pathways underlie the risk of rheumatoid arthritis. Nat Genet. 2017:49:1120-5. 